\title{
The stress-strain state and the microstructure in disk-shaft solid-phase bonds of dissimilar nickel-based alloys
}

\author{
V. A. Valitov ${ }^{1}$, A. Kh. Akhunova ${ }^{1}$, E. V. Galieva ${ }^{1, \dagger}$, S. V. Dmitriev ${ }^{1,2}$, \\ R. Ya. Lutfullin ${ }^{1}$, M. Y. Zhigalova ${ }^{1}$ \\ †Galieva_elvina_v@mail.ru \\ ${ }^{1}$ Institute for Metals Superplasticity Problems of RAS, 39 Khalturin str., 450001, Ufa, Russia \\ ${ }^{2}$ National research Tomsk State University, 36 Lenin Ave., 634050, Tomsk, Russia
}

\begin{abstract}
Finite-element calculations have been carried out to determine the strain distribution formed during welding by a combination of pressure and shear in samples imitating the constituent parts of a "disk-shaft" type gas-turbine engine bimetallic component made of heterophase nickel-based superalloys. A physical simulation of this process has also been carried out. Computer simulations were accomplished using a two-dimensional axysymmetric model by means of DEFORM-2D package. EP975 and EK79 alloys with ultrafine-grained (UFG) structure were chosen as the materials for the disk and shaft, respectively. The initial strain rate was $10^{-5} \mathrm{~s}^{-1}$, and the welding temperature $1100^{\circ} \mathrm{C}$. The behavior of materials was described by experimental curves obtained for EP975 and EK79 alloys under a uniaxial compression at the welding temperature. According to known experimental data, shear strain near the welded surfaces improves the solid-phase bond quality and strength. Additionally, the solid-phase bond quality is enhanced by grain refinement, which leads to an increase of the area of grain boundaries and to accelerated grain boundary diffusion. The results of computer simulations have revealed that in order to increase the area of regions with large shear strain values one has to increase the displacement of the shaft relative to the disk. From physical simulation, it has been concluded that a high quality solid-phase bond formation is promoted by a small shear strain of EK79 and EP975 alloys with UFG structure under superplasticity conditions. Basing on the studies carried out, a possibility of obtaining a "disk-shaft" type part from EP975 and EK79 nickel alloys with a preliminarily processed UFG structure using solid-phase bonding by means of pressure and shear welding has been shown.
\end{abstract}

Keywords: finite-element modeling, pressure welding, nickel-based alloy, deformation, ultrafine-grained structure, superplasticity, hightemperature strength.

\section{Исследование напряженно-деформированного состояния и микроструктуры в зоне твердофазного соединения типа диск-вал из разноименных сплавов на основе никеля}

\author{
Валитов В.А. ${ }^{1}$, Ахунова А.Х. ${ }^{1}$, Галиева Э. В. ${ }^{1 \dagger}$, Дмитриев С. В. ${ }^{1,2}$, \\ Лутфуллин Р. Я. ${ }^{1}$, Жигалова М. Ю. ${ }^{1}$ \\ †Galieva_elvina_v@mail.ru \\ ${ }^{1}$ Институт проблем сверхпластичности металлов РАН, ул. Халтурина 39, 450001, Уфа, Россия \\ ${ }^{2}$ Национальный исследовательский Томский государственный университет, пр. Ленина 36, 634050, Томск Россия \\ Проведен конечно-элементный расчет распределения деформаций, развивающихся в процессе сварки давлением \\ со сдвигом в образцах, имитирующих составные части биметаллической детали газотурбинного двигателя типа \\ «диск-вал» из гетерофазных деформируемых жаропрочных никелевых сплавов. Кроме того, выполнено физическое \\ моделирование данного процесса. Компьютерное моделирование проводили в двумерной постановке (осесимме- \\ тричная задача) с помощью пакета прикладных программ DEFORM-2D. B качестве материала для «диска» и «вала» \\ были выбраны сплавы в ультрамелкозернистом (УМЗ) состоянии ЭП975 и ЭК79, соответственно. Начальная ско- \\ рость деформации составляла $10^{-5} \mathrm{c}^{-1}$, температура сварки $-1100^{\circ} \mathrm{C}$. При расчетном моделировании поведение
}


материала образцов описывалось экспериментальными кривыми, полученными при одноосном сжатии сплавов ЭП975 и ЭК79 при температуре сварки. Согласно известным экспериментальным данным, сдвиговая деформация вблизи свариваемых поверхностей способствует повышению качества твердофазного соединения и его прочности. Также положительно на качество сварного соединения влияет уменьшение размера зерна, приводящее к увеличению протяженности границ зерен и ускорению зернограничной диффузии. Результаты компьютерного моделирования показали, что для увеличения протяженности участков с достаточно большими значениями сдвиговых деформаций (порядка одного процента и выше) целесообразно увеличивать перемещение заготовки вала относительно заготовки диска. Результаты физического эксперимента позволяют заключить, что формированию качественного твердофазного соединения благоприятствует небольшая сдвиговая деформация в условиях сверхпластичности соединяемых сплавов ЭК79 и ЭП975 с УМЗ структурой. На основании проведенных исследований показана возможность получения в процессе сварки давлением неразъемного твердофазного соединения типа «диск-вал» из никелевых сплавов ЭП975 и ЭК79 с предварительно подготовленной ультрамелкозернистой структурой.

Ключевые слова: конечно-элементное моделирование, сварка давлением, никелевый сплав, деформация, ультрамелкозернистая структура, сверхпластичность, жаропрочность.

\section{1. Введение}

В настоящее время актуальными являются исследования, направленные на разработку инновационных технологий получения неразъемных соединений при изготовлении биметаллических деталей типа блиск, диск-диск, диск-вал из жаропрочных никелевых и титановых сплавов с целью повышения надежности и снижения массы роторных конструкций, которые предназначены для газотурбинного двигателя (ГТД) нового 5-го поколения [1-4]. Для получения неразъемных соединений при изготовлении вышеуказанных биметаллических деталей могут быть использованы различные методы сварки и пайки: диффузионная сварка [5-7], высокотемпературная пайка, сварка трением, сварка давлением (СД) и другие методы [8-16].

Традиционные методы сварки плавлением для изготовления биметаллических деталей из современных жаропрочных никелевых сплавов неприменимы из-за высокого содержания в них алюминия. Алюминий способствует высокому содержанию гамма-штрих фазы ( $\gamma^{\prime}$-фазы), которая резко ухудшает свариваемость вследствие высокой скорости ее выделения [17]. Следовательно, в данном случае, применимы только те методы получения твердофазных соединений, в которых минимизировано возникновение фазовых и термических напряжений в процессе формирования твердофазного соединения. Поэтому при соединении жаропрочных сплавов наиболее широко используются методы диффузионной сварки и сварки давлением [5-11].

Для улучшения условий образования физического контакта между свариваемыми поверхностями при СД возможны несколько решений, таких как, предварительная обработка свариваемых поверхностей, нанесение рельефа [18-22], внесение прокладки между контактными поверхностями [23-25], нагрев заготовок, комбинирование СД с различными способами деформации. При этом необходимо учитывать влияние пластической деформации на микроструктуру интерметаллида, а также влияния температурно-скоростных условий на пластическую деформацию [26, 27].

Использование методов СД, в которых присутствует сдвиговая компонента деформации [28-30] в ряде случаев позволяет обеспечить получение беспористого твердофазного соединения с высокими характеристиками механических свойств. В то же время, как показано в работе [31], при линейной сварке трением жаропрочного никелевого сплава ЭП742 зона твердофазного соединения обладает значительной пористостью и неоднородностью, характеризуется образованием грубых выделений карбидов, способствующих возникновению и развитию трещин, что резко ухудшает качество и прочность соединения при вышеуказанной обработке. Эти недостатки могут быть преодолены при сварке давлением (СД) в условиях сверхпластичности (СП) [31], а также в комбинации со сдвиговой компонентой деформации.

В данной работе рассматривается метод получения сварного соединения комбинированием СД со сдвигом, который предполагает внедрение клиновидного вала в диск, что обеспечивает сдвиговую деформацию на их контактной поверхности. Методом компьютерного моделирования рассчитано напряженно-деформированное состояние в данном процессе, что позволило оценить величину сдвиговой деформации на контактной поверхности биметаллического соединения типа дисквал из гетерофазных жаропрочных никелевых сплавов ЭП975 и ЭК79.

\section{2. Конечно-элементное моделирование}

Проводилось моделирование сварки давлением со сдвигом для образцов, имитирующих детали газотурбинного двигателя «диск» и «вал», общий вид которых приведен на рис. 1. «Вал» представлял собой цилиндр диаметром 11 мм и высотой 18 мм, сопряженный с усеченным конусом высотой 12 мм, в котором угол между нижним основанием и образующей составлял $3^{\circ}$. Отверстие в «диске» имело сужение в диаметре с 11 мм до 9,8 мм. Угол наклона внутренней стенки составлял $3^{\circ}$. Общая высота диска - 15 мм.

Расчет проводили для 1/2 образцов в двумерной постановке (осесимметричная задача) с помощью пакета прикладных программ DEFORM-2D. Конечно-элементная модель приведена на рис. 2. Линия АВ являлась осью симметрии модели. Для проведения сварки, «диск» устанавливался на неподвижную траверсу (BC) и в отверстие «диска» полностью помещалась коническая часть «вала». Посредством задания перемещения на подвиж- 
ную траверсу (АВ), осуществлялось внедрение «вала» в «диск». Деформируемые тела разбивали на двадцатиузловые изопараметрические конечные элементы с квадратичной аппроксимацией поля перемещений. Количество элементов «диска» - 2570, «вала» - 3000. Скорость перемещения траверсы составляла $10^{-5} \mathrm{c}^{-1}$. Величина перемещения траверсы $-1,2$ и 3 мм. Сварка проводилась в изотермических условиях при температуре $1100^{\circ} \mathrm{C}$. В качестве материала для «диска» и «вала» были выбраны деформируемые жаропрочные сплавы в ультрамелкозернистом состоянии ЭП975 и ЭК79, соответственно.

При проведении компьютерного моделирования деформирующий инструмент (траверсы) имели свойства абсолютно жесткого тела, деформируемые тела («диск» и «вал») принимались пластичными. Материал образцов описывался экспериментальными кривыми, полученными при одноосном сжатии сплавов ЭП975 и ЭК79 при температуре сварки. Контактные условия на границах пар «траверса-вал» и «диск-вал» описывались моделью трения по Зибелю. Величина коэффициента трения принималась равной 0.3 .

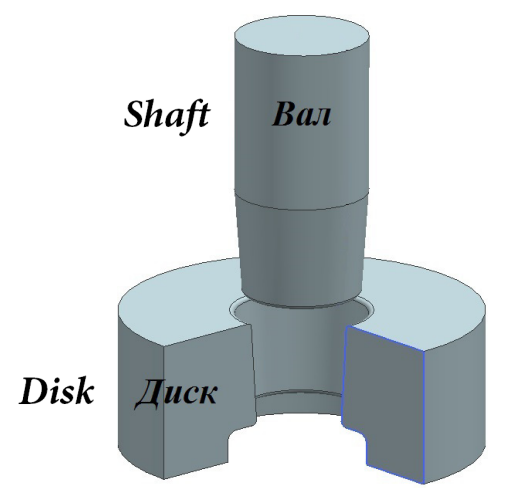

Рис. 1. Общий вид образцов, имитирующих детали газотурбинного двигателя «диск» и «вал». «Диск» дан в разрезе. Fig. 1. The general view of the samples imitating the parts of the gas turbine engine "disk" and "shaft". The "disk" is given in section.

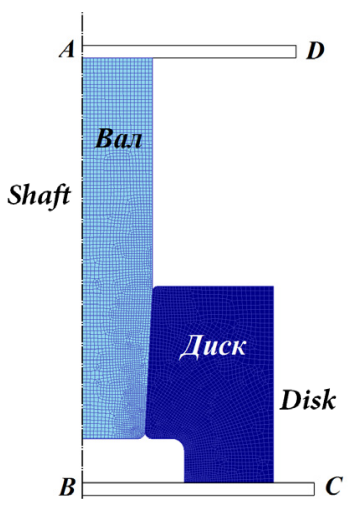

Рис. 2. Конечно-элементная модель детали «диск-вал». Линия $\mathrm{AB}$ является осью симметрии. Перемещение задается через траверсу, обозначенную как AD. Траверса BC неподвижна.

Fig. 2. Finite element model of the «disk-shaft» part. The AB line is the symmetry axis. The deformation is achieved via displacement of the traverse, denoted as AD. Traverse BC is fixed.
При анализе результатов моделирования рассматривалось распределение сдвиговых компонент деформации, поскольку нормальные компоненты деформации слабо влияют на качество сварного соединения. Результаты моделирования в виде картин распределения интенсивности деформаций и сдвиговой деформации представлены на рис. 3 и 4. Данные приведены для величин перемещения траверсы 1, 2 и 3 мм, что составляет, соответственно, 8,17 и 25\% от высоты конической части «вала», которая находится непосредственно в контакте с «диском» (рис. 2).

По картинам распределения интенсивности деформаций (рис. 3) можно заключить, что деформация в цилиндрической части вала несколько больше, чем в зоне контакта с диском. При этом максимальная деформация в этой области не превышает $4 \%$ для перемещения траверсы на 1 мм, 6\% для перемещения на 2 мм и $8 \%$ для перемещения на 3 мм, что является положительным моментом, поскольку общая деформация вала нежелательна. Степень деформации в зоне контакта «вала» и «диска» распределена достаточно равномерно и находится в диапазоне 1-2\% для перемещения траверсы на 1 мм, 3-4\% для перемещения на 2 мм и 4-6\% для перемещения на 3 мм. Картины распределения сдвиговых деформаций (рис. 4) показывают их равномерное распределение в зоне контакта и лежат в диапазоне $0,75-1,5 \%$ для перемещения траверсы на 1 мм, 1,5-3\% для перемещения на 2 мм и 2,3-3\% для перемещения на 3 мм. Таким образом, даже при небольшой деформации самого «вала», можно достичь достаточной деформации в зоне контакта «вала» и «диска».

Как показали результаты физического эксперимента, приведенные в следующем разделе, перемещение траверсы на 1 мм, для которого значения степени деформации и сдвиговых деформаций в зоне контакта «вала» и «диска» достигают 1-2\%, является достаточным для получения качественного ТФС, благодаря отсутствию пор и выделений избыточных фаз, что было показано при исследовании микроструктуры сварных образцов с использованием метода сканирующей электронной микроскопии.

\section{3. Физическое моделирование}

Был проведен физический эксперимент по сварке давлением со сдвигом образцов из сплавов ЭП975 и ЭК79, имитирующих поведение диска и вала, соответственно (рис. 5, 6). Скорость деформации составляла $10^{-5} \mathrm{c}^{-1}$. Температура сварки $-1100^{\circ} \mathrm{C}$. Величина перемещения траверсы - 1 мм.

При металлографическом анализе зоны ТФС дисквал обнаружено неравномерное распределение пористости вдоль конической части вала. В периферийных зонах ТФС диска с валом то есть, зон вблизи верхнего и нижнего оснований диска (рис. 5а, поз. 1, 3), относительная протяженность пор составляет не более $15 \%$. В центральной зоне (рис. 5а, поз. 2), соответствующей половине высоты конической части вала, относительная протяженность пор составляет не более 2\%. При микрорентгеноспектральном анализе установлено, что в зоне 


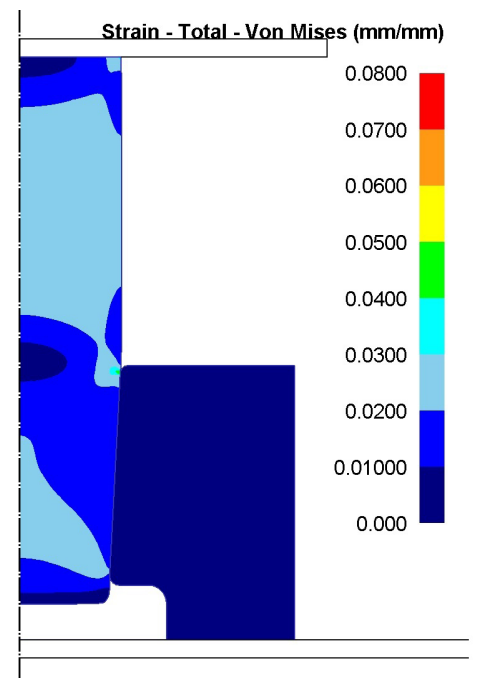

a

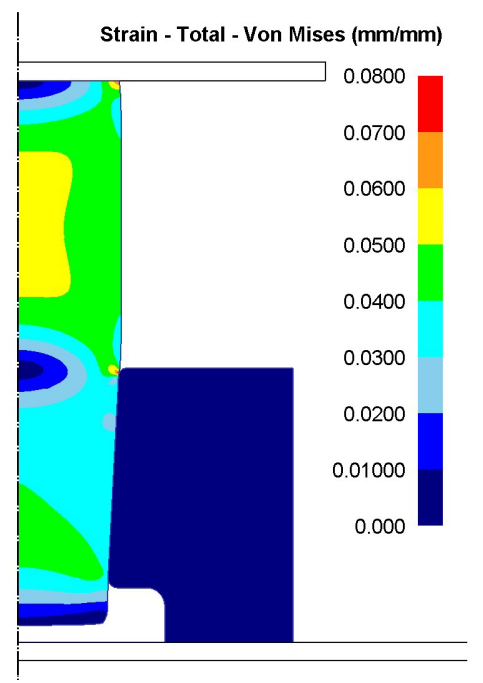

b

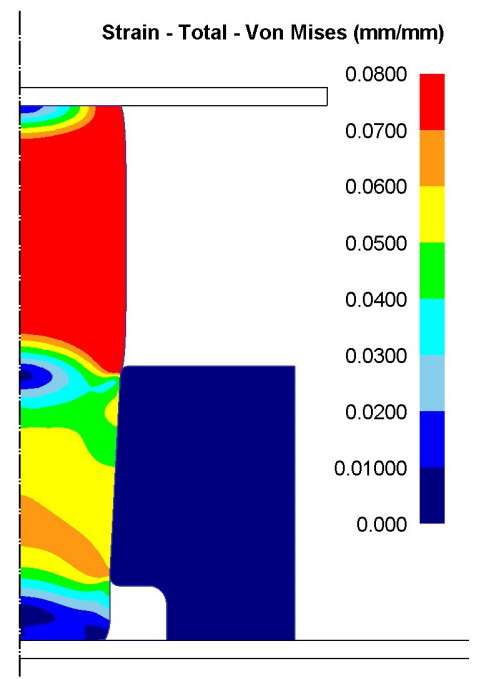

C

Рис. 3. Распределение интенсивности деформаций для величин перемещения траверсы: a) 1 мм, b) 2 мм, с) 3 мм. Fig. 3. Distribution of the deformations intensity for the values of the traverse displacement: a) $1 \mathrm{~mm}$, b) $2 \mathrm{~mm}$, c) $3 \mathrm{~mm}$.

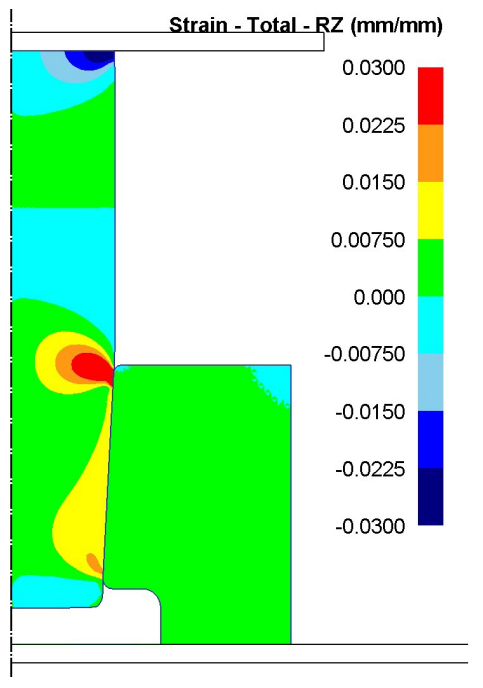

a

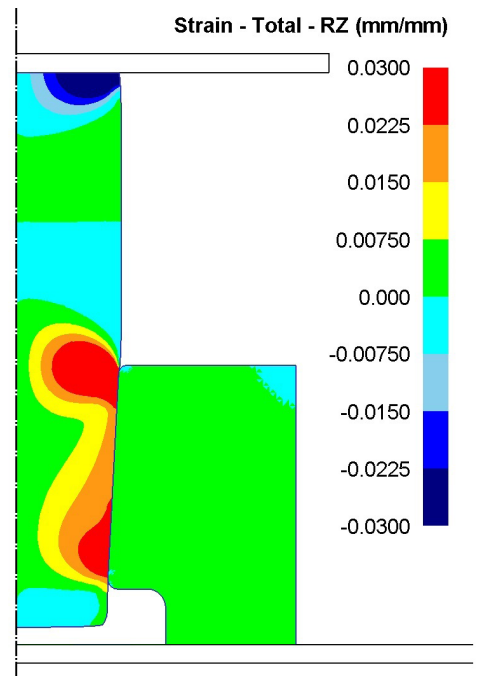

b

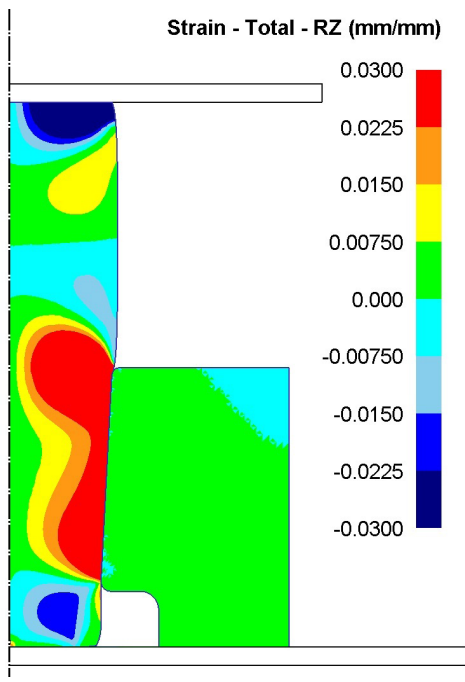

c

Pис. 4. Распределение сдвиговой деформации для величин перемещения траверсы: a) 1 мм, b) 2 мм, с) 3 мм.

Fig. 4. Distribution of shear deformation for the values of traverse displacement: a) $1 \mathrm{~mm}, \mathrm{~b}) 2 \mathrm{~mm}, \mathrm{c}) 3 \mathrm{~mm}$.
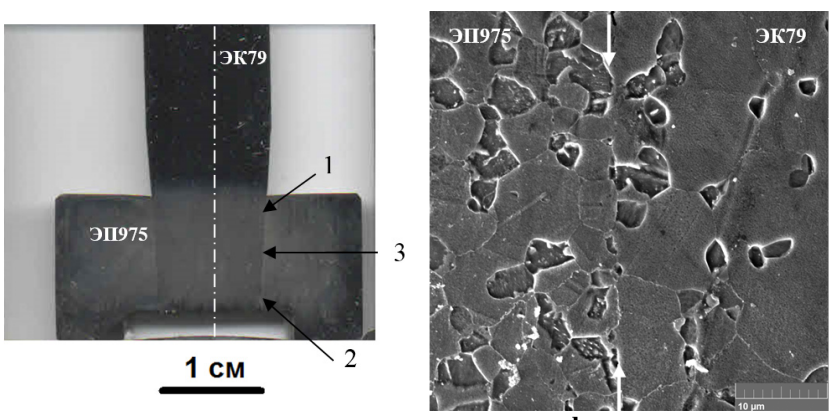

a

b

Рис. 5. Результаты физического моделирования. Образцы после сварки давлением: а) общий вид; b) структура в зоне твердофазного соединения.

Fig. 5. The results of the physical modelling. Pressure welding samples: a) general view; b) structure in the solid-phase bond zone.
ТФС наблюдается монотонное изменение состава легирующих элемнтов (рис. 6). По результатам измерения микротвердости обнаружено, что ее величина в зоне соединения равна значению микротвердости соединяемых материалов, в предлах погрешности (рис. 7.)

По всей видимости, формированию твердофазного соединения из разноименных никелевых сплавов ЭК79 и ЭП975 благоприятствуют не только температурноскоростные условия сверхпластической деформации соединяемых материалов, но и сдвиговая деформация, которая по данным конечно-элементного моделирования составляет 8,3\%, возникающая вследствие осевого смещения «вала» относительно «диска». При этом, важно отметить, что в процессе сдвиговой деформации возможно механическое разрушение оксидной пленки на контактных поверхностях соединяемых материалов и образование ювенильных поверхностей, что, как показано в работе [32], необходимо для формирования неразъемного беспористого твердофазного соединения. 


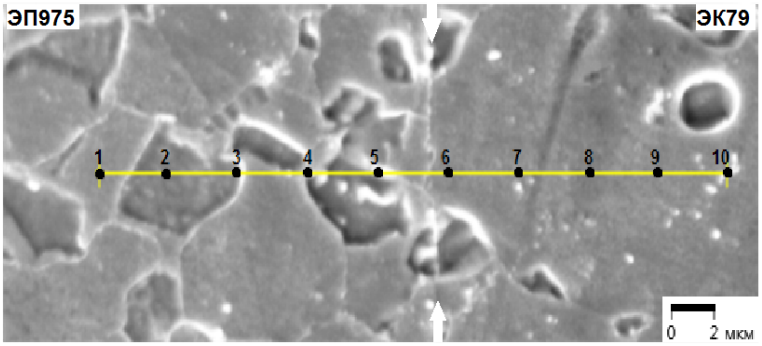

ТФС ЭП975+ЭК79

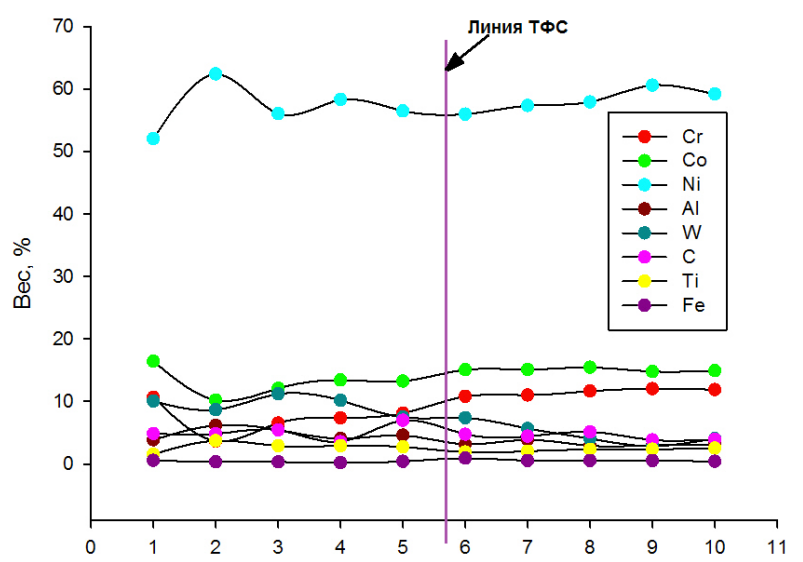

Рис. 6. Микрорентгеноспектральный анализ зоны соединения в сочетании ЭП 975 + ЭК79.

Fig. 6. Solid-phase bonding X-ray microanalysis of EP975 + EK79.

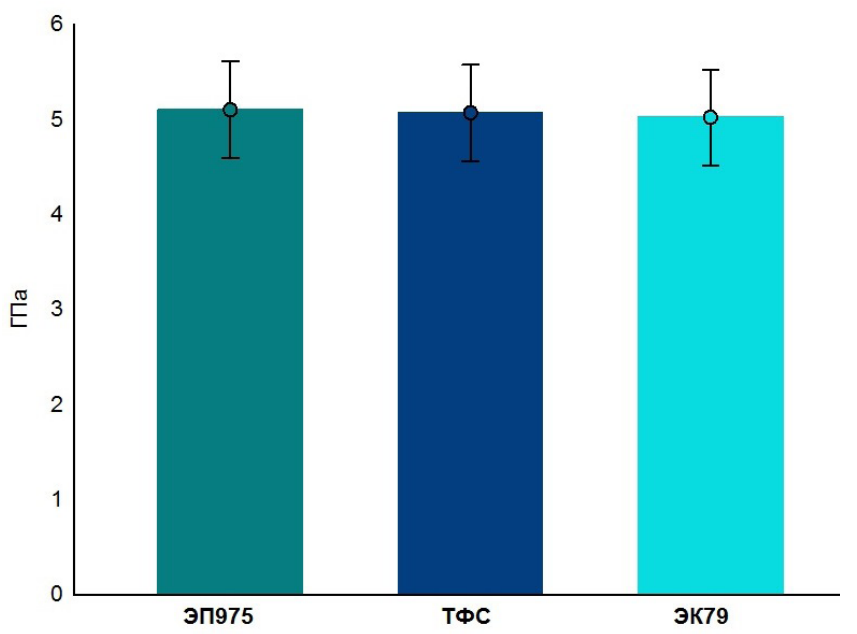

Рис. 7. Микротвердость зоны ТФС ЭК79 + ЭП975.

Fig. 7. Microhardness of the EK79 + EP975 solid-phase bond.

\section{4. Выводы}

1) Для получения неразъемного соединения типа «дисквал» из жаропрочных никелевых сплавов ЭП975 и ЭК79 предложен метод сварки давлением путем внедрения клиновидного вала в диск. Данный метод обеспечивает сдвиговую деформацию на контактной поверхности, что улучшает условия для создания физического контакта двух свариваемых деталей.

2) С применением компьютерного моделирования установлено, что степень деформации и сдвиговые деформации в зоне контакта «вала» и «диска» распределены достаточно равномерно и находятся в интервале $2-6 \%$ и 1,5-3\%, соответственно, при осевом смещении «вала» относительно «диска» на $8,3-25 \%$ от значений высоты конической части «вала».

3) На основе результатов физического моделирования показано, что осевое смещение «вала» относительно «диска» на 8,3\% от высоты конической части «вала», обеспечивает формирование беспористого твердофазного соединения с отсутствием выделений избыточных фаз.

Благодарность/Acknowledgements. Работа выполнена при финансовой поддержке гранта РФФИ № 14-08-97061 p_поволжье_а

\section{Литература/References}

1. A. V. Logunov, Yu. N. Shomotin. Science and Technology (2013) 256 p. (in Russian) [А. В. Логунов, Ю. Н. Шмотин Москва: ООО «Наука и технология». 2013. 256 с.]

2. Tresa M. Pollock. Sammy Tin. J. propul. power. 22 (2006) $361-374$.

3. Heather J. Sharpe, Ashok Saxena. Adv. Mat. Res. 278 (2011) 259-264.

4. D. U. Furrer, R. Shankar, C. White. Journal of Metals (JOM-J Met) (2003) 32-34.

5. A.V. Lyushinsky, Ye.V. Nikolich, A.A. Zhloba, S. V. Kharkovsky, A. V. Borovsky, D. S. Karyaka. Welding International, 29 (2015), $394-396$.

6. A. A. Shirzadi, E. R. Wallach. Sci. Technol. Weld. Joi. 9 (2004) $37-40$.

7. N.P. Wikstrom, A.T. Egbewande, O.A. Ojo. J. Alloy Compd. 460 (2008) 379-385.

8. A.V. Lushinskii Part1. Svarochnoe proizvodstvo (7), 17 - 22 (2016). (in Russian) [А. В Люшинский. Часть 1) // Сварочное Производство, № 7, 2016, с. 17 -22.]

9. A. A. Shirzadi and E. R Wallach. Science and Technology of Welding and Joining. 9 (1), $37-40$ (2004).

10. Jiakun Liu, Jian Cao, Xingtao Lin, Xiaoguo Song, Jicai Feng. Materials and Design 49 (2013) 622-626.

11. K. B. Povarova, A. A. Drozdov, V. A. Valitov, E. V. Valitova, S.V. Ovsepyan, O.A. Bazyleva. Russian metallurgy (Metally). 2014 (9) 733-741 (2014). (in Russian). [К.Б. Поварова, В.А. Валитов, С.В. Овсепян, А.А. Дроздов, О.А. Базылева, Э.В. Валитова Металлы. (5), 61 - 70 (2014).

12. A. V.Skugoreev, A. N. Afanasiev-Khodykin, A. M. Rogalev, D. S. Lozhkova. Technology of light alloys. (3), 75-82 (2016). (in Russian) [А.В. Скугорев, А.Н. АфанасьевХодыкин, А. М. Рогалев, Д.С. Ложкова. Технология легких сплавов. 2016, № 3, С. 75 - 82.]

13. V. I. Lukin, V. S. Rylnikov, O. A. Bazyleva, A. N. AfanasievKhodykin. Svarochnoe proizvodstvo. (6), 15-18 
(2014). (in Russian) [В.И. Лукин, В.С. Рыльников, О.А. Базылева, А.Н. Афанасьев-Ходыкин // Сварочное производство, 2014 г., № 6, С. 15 - 18.]

14. A.Kh. Akhunova, A.I. Pshenichnyuk, S. V. Dmitriev, A.R. Safiullin, R.V. Safiullin. Russian metallurgy (Metally) (7), 33 - 38 (2013). (in Russian) [А. Х. Ахунова, А.И. Пшеничнюк, С.В. Дмитриев, А.Р. Сафиуллин, Р.В. Сафиуллин. Деформация и разрушение материалов. (7), 33 - 38 (2013).].

15. A.Kh. Akhunova, E.V. Galieva, K.B. Povarova, O. A. Bazyleva, V. A. Valitov, S. V. Dmitriev, A. A. Drozdov, E.G. Arginbaeva. Fundamentalnye problem sovremennogo materialovedeniya. 13 (1), 131-135 (2016). (in Russian) [Ахунова А.Х., Галиева Э.В., Поварова К.Б., Базылева О.А., Валитов В.А., Дмитриев С. В., Дроздов А.А., Аргинбаева Э.Г. Фунд аментальные проблемы современного материаловедения. 13 (1), 131 - 135 (2016).].

16. A. R. Safiullin, R. V. Safiullin, F. F. Safin, A. Kh. Akhunova, S.V. Dmitriev. Perspectivnye materialy. (15), 114-118 (2013). (in Russian) [А. Р. Сафиуллин, Р. В. Сафиуллин, Ф.Ф. Сафин, А.Х. Ахунова, С.В. Дмитриев. Перспективные материалы. 15, 114 - 118 (2013).]

17. S.V. Kishkin Creation, research and application of high-temperature alloys: selected works (To the 100th anniversary of his birth) M. Science. (2006) 407 p. (in Russian) [C.T. Кишкин Создание, исследование и применение жаропрочных сплавов: избранные труды (К 100-летию со дня рождения). - М.: Наука, 2006. - 407c.]

18. A.Kh. Akhunova, E.V. Valitova, S.V. Dmitriev, V.A. Valitov, R. Ya. Lutfullin. Welding International. 30 (6), $488-491$ (2016).

19. A.Kh. Akhunova, S.V. Dmitriev, V.A. Valitov, E. V. Valitova. Russian metallurgy (Metally) (11), 13 - 17 (2014). (in Russian) [А.Х. Ахунова, С.В. Дмитриев, В. А. Валитов, Э. В. Валитова Деформация и разрушение материалов. (11), 13 - 17 (2014).].

20. A. Kh. Akhunova, S.V. Dmitriev, E.V. Valitova, V.A. Valitov. Fundamentalnye problem sovremennogo materialovedeniya. 11 (2), 159-162 (2014). (in Russian) [А.Х. Ахунова, С.В. Дмитриев, Э.В. Валитова, В.А. Валитов. Фундаментальные проблемы современного материаловедения. 11 (2), 159 - 162 (2014).]

21. A.Kh. Akhunova, E.V. Galieva, A.A. Drozdov,
E. G. Arginbaeva, S. V. Dmitriev, R. Ya. Lutfullin. Letters on Materials. 6 (3), 210-215 (2016). (in Russian) [А.Х. Ахунова, Э. В. Галиева, А.А. Дроздов, Э.Г. Аргинбаева, С. В. Дмитриев, Р.Я. Лутфуллин. Письма о материалах. 6 (3), 210 - 215 (2016).

22. V.A. Valitov., K.B. Povarova, O.A. Bazyleva, A. A. Drozdov, S. V. Ovsepyan, E. V. Galieva. Materials Science Forum 838 - 839, 523 - 527 (2016).

23. E.V. Valitova, A.Kh. Akhunova, V.A. Valitov, S. V. Dmitriev, R. Ya. Lutfullin, Letters on Materials. 4 (3), $190-194$ (2014). (in Russian) [Э.В.Валитова, А.Х. Ахунова, В.А. Валитов, С.В. Дмитриев, Р.Я. Лутфуллин, М.Х. Мухаметрахимов. Письма о материалах. 4 (3), 190 - 194 (2014)].

24. A.Kh. Akhunova, S.V. Dmitriev, E.V. Galieva, V.A. Valitov. Fundamentalnye problem sovremennogo materialovedeniya. 12 (3), 289-292 (2015). (in Russian) [А.Х. Ахунова, С.В. Дмитриев, Э.В. Галиева, В.А. Валитов. Фундаментальные проблемы современного материаловедения. 12 (3), 289 - 292 (2015).]

25. E. V. Valitova, R. Ya.Lutfullin, M. Kh. Mukhametrakhimov, V. A. Valitov, A. Kh. Akhunova, S. V. Dmitriev. Letters on Materials. 4 (4), 291 - 294 (2014).

26. I. I. Musabirov, I. M. Safarov, M. I. Nagimov, I.Z. Sharipov, R. R. Mulyukov, V. V. Koledov, A. V. Mashirov, A. I. Rudskoi. Physics of the Solid State. 58 (8), 1605 - 1610 (2016).

27. I.I. Musabirov, I.M. Safarov, R.R. Mulyukov, I.Z. Sharipov, V. V. Koledov. Letters on Materials. 4 (4), 265 - 268 (2014). (in Russian)

28. V.I. Lukin, V.G. Kovalchuk, M.L. Samorukov, Yu. M. Gidnev. Vestnik BMSTU 114-121 (2011) (In Russian) [В. И. Лукин, В. Г. Ковальчук, М. Л. Саморуков, Ю.М. Гриднев. Вестник МГТУ им. Н.Э. Баумана. $114-121(2011)]$

29. O.T. Ola, O.A. Ojo, P. Wanjara and M.C. Chaturvedi. Metallurgical and Materials Transactions. 43A (2012), $921-933$.

30. W. T. Chandler, A. K. Ghosh and W. M. Mahoney. Journal of Spacecraft and Rockets, 21 (1), 61-64, (1984).

31. V.M. Bichkov, A/S. Selivanov, A. Yu. Medvedev and others. Vestnik USATU 16 (7), 112 -116 (2012). (In Russian) [В. М. Бычков, А. С. Селиванов, А. Ю. Медведев и др. Вестник УГАГУ. 16 (7), 112 - 116 (2012).]

32. Patent RF № 2608118, 13.01.2017 (in Russian) [Патент РФ № 2608118, 13.01.2017]. 